\title{
SCADA Based Automatic Direct Reduction of Iron Process using Allen-Bradley Programmable Logic Controllers
}

\author{
Mr.G.Venkateswarlu \\ CVR College of Engineering, Department of EIE., Hyderabad, India \\ Email: venkigummadilli@gmail.com
}

\begin{abstract}
In this paper, we propose an SCADA Based Automatic Direct Reduction of Iron (DRI) Process Using Allen-Bradley Programmable Logic Controllers. SCADA stands for Supervisory Control and Data Acquisition system. The main objective of the project is to get the high grade (high quality) of Sponge Iron. Main quality of the product depends of the temperature of the different junctions of the rotary KILN .Initially, measures the temperatures of the junctions of KILN and fed as inputs to the PLC which in turn sent to PC through RS-232 for necessary processing. A PLC program is developed based on the signals received from the PLC analog input module, process the signals and send the output signals to the PLC output modules, which is programmed to control the fuel conveyor and shell air fan by using PLC-PID controller functions. Here controller parameters can be determined by PID-tuning methods.PLC program can be implemented by considering Rotary KILN direct reduction of iron process.
\end{abstract}

The SCADA screens are developed on PC regarding process going in rotary KILN using RS-VIEW SCADA software. The Allen Bradley micrologix 1200 PLC have to be linked up with the supporting SCADA RS-VIEW by giving necessary tag names. With the help of SCADA, simulation of the physical equipment is also developed to monitor the quantities to be measured and status of the in the plant from field point.

Index Terms: DRI process, Analog I/O Modules, Rotary KILN, PLC and SCADA.

\section{INTRODUCTION}

DRI process: Large amount of sponge iron is produced in INDIA only and no. of sources are available in INDIA to get raw material iron ore and we are exporting raw material iron to China also.

Direct reduction of iron is the process of making the steel or iron product .Initially product can be produced by Direct reduction of iron process, This is also called as sponge iron .Initially this can be processed in another way, which is called as pig iron[1]. In this process iron ore can be reduced into lumps and pellets by using reducing gases. This gas can be produced from fuels like coal or gas. Total reduction process is going into the KILN. It is also called as reactor or furnace or rotary KILN. In this process hydrogen and carbon monoxide are the major gases of reducing gas. Here raw material iron ore is fed to KILN along with the coal and dolomite. Dolomite reduces the absorption of sulfur from the coal while reduction process is going.

In this process mainly have seven junctions; each junction takes different chemical reactions in order to produce desired grad of iron .Basically, we have to provide oxygen to produce satisfactory combustion process in the rotary KILN. It takes these chemical reactions b/w $750^{\circ} \mathrm{c}$ and $1100^{\circ} \mathrm{c}$ temperature levels .Sponge iron plants give good grade of iron products. These plants were established throughout the world. In this Paper rotary KILN junction temperatures can be controlled by adjusting the speed of conveyor belt. Once the speed is controlled then the flow of fuel will be controlled, once the flow of fuel is controlled then the temperature will be controlled and then reduction of iron will be controlled [1]. Here quality of product mainly depends on temperature of gas or breath and the chamber pressure; these parameters can be automated by using PLC and SCADA system.

\section{Block Diagram Of The APPLiCation}

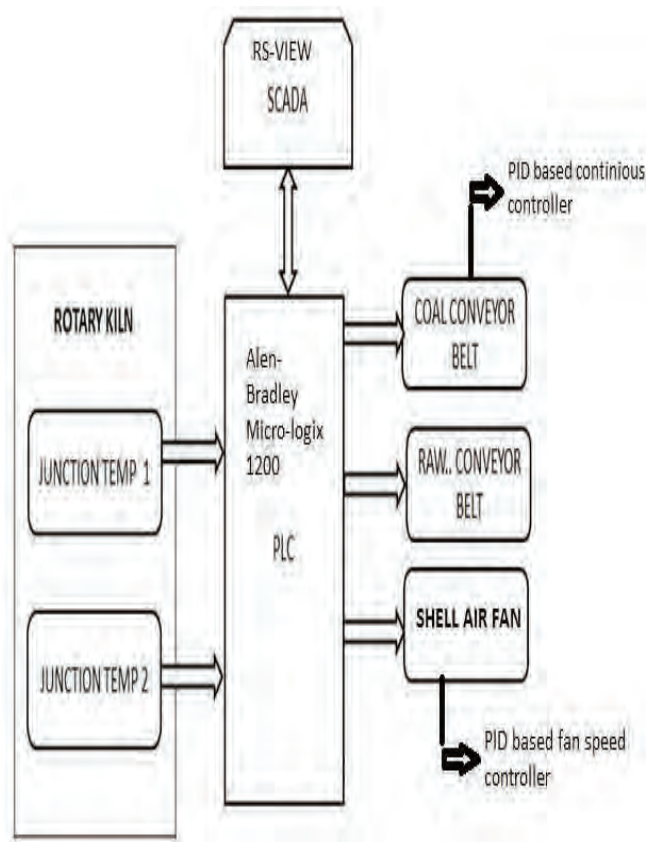

Fig 1.Block diagram

The block diagram Fig.1describes the interfacing of hardware and software i.e., interfacing of input and output field devices with programmable logic controllers. Eight 
input field devices are interfaced with the PLC input output modules. In this project eight digital input terminals, two analog input terminals, one digital output terminal and two analog output terminals are used. Eight digital input terminals are used to interface eight starts and stop buttons for on and off of coal conveyor belt, total system on and off switches and Raw material Iron ore on and off switches [2]. These switches are named as sw1, sw2, sw3, sw4, sw5, sw6, sw7 and sw8.Before going to make the connection of field devices ,power supply should be provided to input and output modules of PLC, different PLC's requires different power supply rating, this PLC requires 24 VDC power supply. This will be provided externally (or) some PLC's have in built power supply unit. Depending on the type of application the switches have to be selected like push buttons (or) limit switches (or) toggle switches.

PLC analog modules are required to interface analog measuring devices like thermocouples. In this project we are using K-type thermocouples, which measures temperature from "-200 c to $1300 \mathrm{c}$ " and produces m volts range of signals [3]. These sensors are interfaced to PLC analog input thermals. In this project we are using two thermocouples to measure the temperature at two different junctions of Rotary KILN but to make interface of thermocouples, which requires signal conditioning circuits to meet the desired specifications of PLC analog input modules. This modules has inbuilt analog to digital converters. So no need to use external ADC devices.

Three output module terminals are required to interface output field devices. These devices are named as Dcm1, Dcm2 and Dcm3 [3]. One output terminal is digital terminals and two more terminals are analog output terminals. Here these motors can't be interfaced directly to the output to the terminals of PLC, which requires isolation (or) drivers circuit like Relays, switches.....etc. mostly analog modules requires power amplifier circuits, which are designed with the power electronics devices. Like $\mathrm{SCR}$, thyristers devices [6]. If the DC motor range is $12 \mathrm{~V}$ then the circuit will be designed with electronic devices like diodes, transistors, resistors...etc.

Here output devices will be controlled by the PLC solid state equipment. The speed of the conveyor motor depends on the junction temperatures at the KILN. We need to develop the program for PID controller by using PLC -PID functions in order to get desired control action on conveyor motor [4]. If the temperature of the Rotary KILN exceeds the tolerance value (or) desired value then PID controller sends the control signals to the fuel conveyor motor unit to get the desired temperature at the junctions of KILN. Here the quality of the product depends on the maintenance of junction temperatures.

\section{I/O DEVICES, KILN AND SPECIFICATIONS}

The devices which are interfaced from the field of industry to PLC input and output terminals are called as field devices which are like switches, sensors, motors and lights .The following devices are interfaced with PLC I/O terminals.
Rotary-KILN: A Rotary-kiln is temperature processing unit is shown below Fig.2. Rotary KILN is also used in cement and pharmacy industries for combustion process, which is driven by PLC through variable frequency drivers. This is acting as a furnace, three raw materials are fed to KILN, and these are iron ore, coal and dolomite. KILN is designed with protected seven layers so temperature cannot be radiated into the environment. Oxygen gas is supplied to KILN to get combustion process. Which is operated for $600^{\circ} \mathrm{c}$ to $1200^{\circ} \mathrm{c}$ while the KILN is rotating then slowly raw material moves down towards the lower end while raw material is fed into the upper end of rotary KILN. Exhausted pressure is released through inlet and outlet doors. Materials produced using rotary kilns include Cement, Lime, Refractory's, Met kaolin, Titanium dioxide, Alumina Vermiculite, Iron ore pellets.

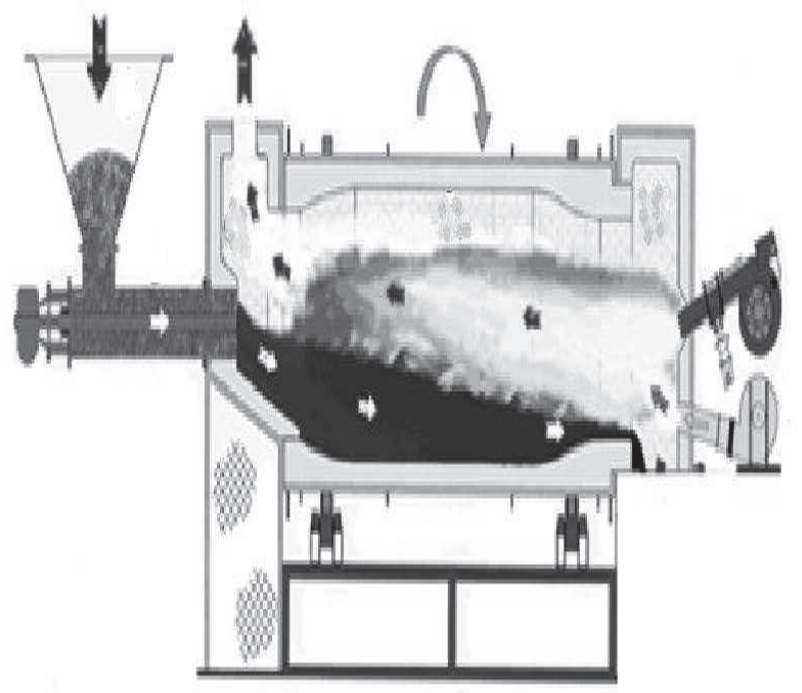

Fig.2 Rotary KILN for DRI process

A typical 500 TPD capacity plant Kiln is $80 \mathrm{~m}$ in length and having $4.34 \mathrm{~m}$ inner $\& 4.85$ outer diameter. The basic components of a rotary kiln are the shell, the refractory lining, support tyres and rollers, drive gear and internal heat exchangers. There are some important components of Rotary kiln.

Reduction process: Six chemical reactions are going through seven junctions of rotary KILN, in the first reaction it produces $2 \mathrm{Feo}$, in the second reaction produces $\mathrm{Fe}$ and the final reaction produces satisfactory product 2 Feo along with the carbon dioxide. The reactions are taken into the KILN is shown in Table. 1 


\begin{tabular}{|c|c|c|c|c|c|c|}
\hline $\begin{array}{l}\text { Si. } \\
\text { No. }\end{array}$ & Reaction & $\Delta \mathrm{G}_{\mathrm{T}}^{0}$ & $\Delta \mathrm{G}_{298}^{0}$ & $\Delta \mathrm{G}_{1273}^{0}$ & $\Delta \mathrm{H}_{298}^{0}$ & $\Delta \mathrm{H}_{1273}^{0}$ \\
\hline 1 & $\mathrm{Fe}_{2} \mathrm{O}_{3}+\mathrm{CO} \rightarrow 2 \mathrm{FeO}+\mathrm{CO}_{2}$ & $\begin{array}{l}+2,120- \\
10.39 \mathrm{~T}\end{array}$ & -976 & $-11,106$ & $+2,270$ & $-2,532$ \\
\hline 2 & $\mathrm{FeO}+\mathrm{CO} \rightarrow \mathrm{Fe}+\mathrm{CO}_{2}$ & $\begin{array}{l}-4,190 \\
+5.13 \mathrm{~T}\end{array}$ & $-2,661$ & $+2,340$ & $-4,430$ & $-5,808$ \\
\hline 3 & $\mathrm{C}+\mathrm{CO}_{2} \rightarrow 2 \mathrm{CO}$ & $\begin{array}{l}-94,200- \\
0.2 \mathrm{~T}\end{array}$ & $-94,260$ & $-94,455$ & $-94,050$ & $-94,561$ \\
\hline 4 & $2 \mathrm{CO}+\mathrm{O} 2 \rightarrow 2 \mathrm{CO} 2$ & $\begin{array}{l}+40,800- \\
41.70 \mathrm{~T}\end{array}$ & $+28,373$ & $-12,284$ & $+41,210$ & $+39,964$ \\
\hline 5 & $\mathrm{FeO}+\mathrm{C} \rightarrow \mathrm{Fe}+\mathrm{CO}$ & $\begin{array}{l}+36,610- \\
36.57 \mathrm{~T}\end{array}$ & $+25,712$ & $-9,944$ & $+36,780$ & $+34,277$ \\
\hline 6 & $\begin{array}{l}\mathrm{Fe}_{2} \mathrm{O}_{3}+3 / 2 \mathrm{C} \rightarrow 2 \mathrm{Fe}+ \\
3 / 2 \mathrm{CO}_{2}\end{array}$ & $\begin{array}{l}54,940- \\
62.68 \mathrm{~T}\end{array}$ & $+36,261$ & $-24,852$ & $+55,225$ & $+50,573$ \\
\hline
\end{tabular}

Table1.Thermodynamic data of important reactions. $\Delta G \& \Delta T$ in $k C a l / K g$.mole, $T$ in oK

Kiln \& conveyor driving motor: In this paper, $12 \mathrm{~V} \mathrm{DC}$ motor has been used to run the KILN, conveyor belt \& Blower fan is shown in fig.3 but in real time all the industries uses three phase induction motors with high power specifications. Specifications of the KILN driving motor (prototype) is shown below table.2.

\begin{tabular}{|l|l|l|}
\hline S.No & parameter & specifications \\
\hline 1 & Lubrication & $\begin{array}{l}\text { synthetic, lifetime } \\
\text { lubricated }\end{array}$ \\
\hline 2 & Wire terminations & $1 / 4^{\prime \prime}$ strip \\
\hline 3 & Efficiency & up to $75 \%$ \\
\hline 4 & Shaft & $\begin{array}{l}1137-1144 \mathrm{crs} \text { (standard) } \\
300 / 400 \text { series }\end{array}$ \\
\hline 5 & Brush life & up to 2,000 hours \\
\hline 6 & Bearings & $\begin{array}{l}\text { pm iron } \text { (standard }) \quad \text { pm } \\
\text { bronze }\end{array}$ \\
\hline 7 & Insulation & class b $\left(130^{\circ}\right.$ c) \\
\hline 8 & Voltage rating & $6-48$ volts dc nominal \\
\hline 9 & Leads & $16 g a$ awg type gpt $\left(80^{\circ} \mathrm{c}\right)$ \\
\hline 10 & Rotation & cw, or reversible \\
\hline
\end{tabular}

Table.2.KILN driving motor specifications

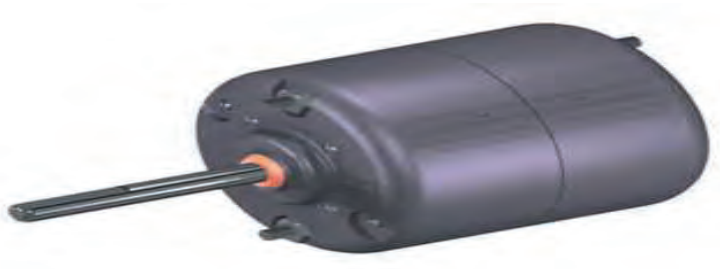

Fig.3 Electrical DC motor

Power Amplifier Circuit: Power amplifier circuits are required to drive a DC motor. This circuit can be shown in Fig.4.This produces proportional amplified signal in order to drive the motor. This signal will be generated by PID controller [5].

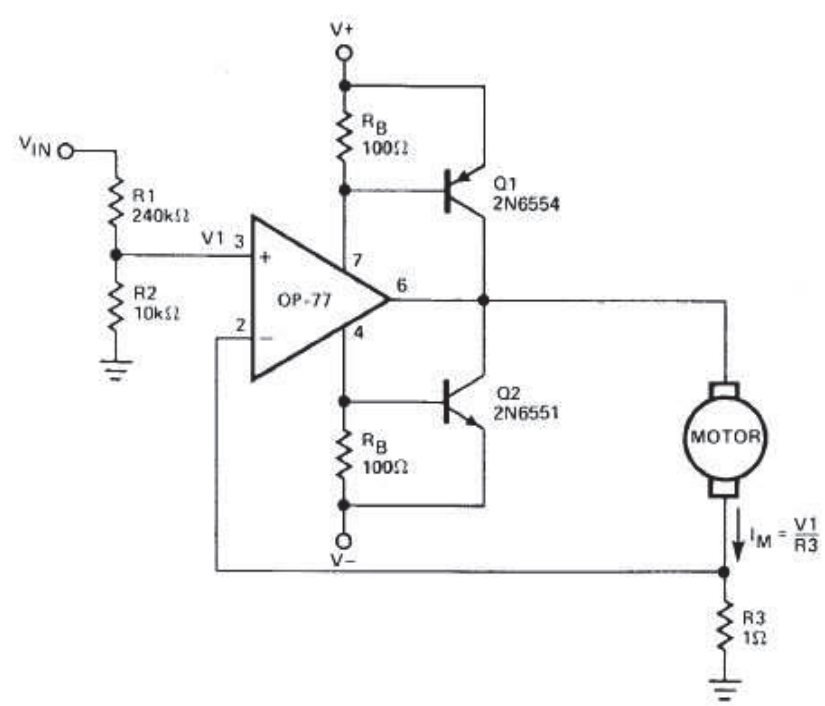

Fig.4 power amplifier circuit

Using a simple operational amplifier and some other common electronic components can be designed a very simple DC motor driver that can be used for a $200 \mathrm{~mA}$ motor application, which is shown in Fig.4.Rb sets the bias point for transistors $\mathrm{Q} 1$ and $\mathrm{Q} 2$. Because Vbe $(\mathrm{ON})$ varies greatly with temperature, a guard band is required to prevent Q1 and Q2 from conducting simultaneously. RB should be selected such that the transistors do not conduct until $\mathrm{lM}$ equals the op amp quiescent supply current, Isy. The transistors will begin to conduct at about Vbe (on) $=$ $0.5 \mathrm{~V}$.

In this circuit:

$$
\begin{aligned}
\mathrm{RB}=[\text { Vbe } & (\text { on }) /(1 \mathrm{SY}+\mathrm{Im})] \\
& =0.5 /(0.0025+0.0025) \\
& =100 \mathrm{Ohms} .
\end{aligned}
$$

To maximize voltage swing across the motor, V1 must be minimized. If at full load $\mathrm{V} 1=0.2 \mathrm{~V}$ with $\mathrm{V}+=$ $15 \mathrm{~V}$ and $\mathrm{V}_{\mathrm{BE} 1}=0.8 \mathrm{~V}$, the voltage across the motor will be:

$$
\begin{aligned}
\mathrm{V}_{\mathrm{M}}=(\mathrm{V}+ & -2)-\mathrm{V}_{\mathrm{BE} 1}-\mathrm{V}_{1} \\
& =(15-2)-0.8-0.2 \\
& =12.0 \mathrm{~V}
\end{aligned}
$$

Vin may be scaled with a resistive divider as:

$\mathrm{V}_{\text {IN }}=(\mathrm{R} 1+\mathrm{R} 2) / \mathrm{R} 2$.

With $\mathrm{R} 1=240 \mathrm{k}$ Ohms and $\mathrm{R} 2=10 \mathrm{k}$ Ohms, $\mathrm{V}_{\mathrm{IN}}=5$ volts, $1_{\mathrm{M}}=200 \mathrm{~mA}$. 


\section{Programmable logic Controller}

In this paper control operations can be done by PLC. This is used for continuous control applications like DC motor speed control, boiler liquid level control. In view of this PLC analog modules are required to interface electrical motors through power amplifier circuits. In early 1960's most of the industries faced problems in trouble shooting of relay circuits. Once the relay was failed then it takes lot of time to find where the problem occurred. If the production need to be increased then it causes to complexity of wiring through relay contacts .In view of this 1969 Richard Morley introduce programmable logic controllers [6]. PLC is solid state equipment, which was mainly introduced for industrial applications and also for home control applications and which performs logical control actions.PLC programming can be written in different alternative programming formats [7], these are ladder programming, functional block and instruction list.

\section{A. MicroLogix 1200}

MicroLogix 1200 expansion I/O (Bulletin 1762) is used to provide discrete and analog inputs and outputs, and specialty modules shown in below fig.5. For the MicroLogix 1200, you can attach up to six additional I/O modules [7]. The number o1762 I/O modules that can be attached to the MicroLogix 1200 is dependent on the amount of power required by the I/O modules

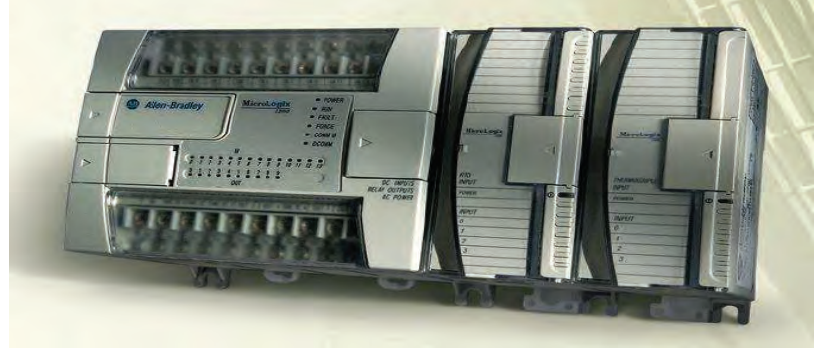

Fig.5 A-B micro logic 1200 PLC

\section{B. Analog I/O Modules of PLC}

Analog I/O modules are used to interface analog input signals from the different sensor like Thermocouples, Strain gauges, flow meters and level sensors, which produces analog signals in the form of mvolts or mAmps. These signals will be amplified and given to PLC analog input terminals and then go for scaling process [7]. In this project thermocouple are used to measure the temperature.

\section{Scaling of analog I/O signals}

After interfacing of input signals to the PLC input terminals, the signal to be scald into desired values in order to match the PLC instruction parameters. PLC has a programming function to make scaling. Through that functions signal can be scaled into desired parameters [7]. In this paper, Micro logic 1200 PLC is used with compact I/O analog modules (1769-IF4 and 1769 OF2). After scaling the parameters to be included to PID setup is shown in Fig.6

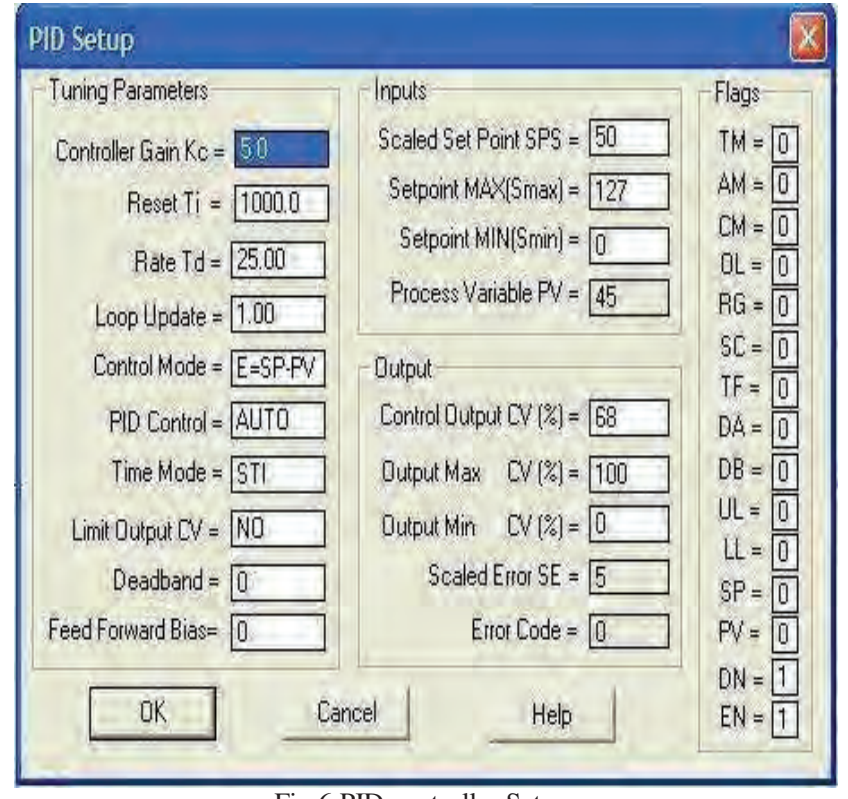

Fig.6.PID controller Setup.

The IF4 has 4 input channels, which are individually configurable (shown in below fig.7). In this example, analog input 0 is configured for 0 to $10 \mathrm{~V}$ and is scaled in engineering units. Word 0 is not being used in a PID instruction. Input 1 (word 1) is configured for 4 to $20 \mathrm{~mA}$ operation with scaling configured for a PID instruction. This configures the analog data for the PID instruction.

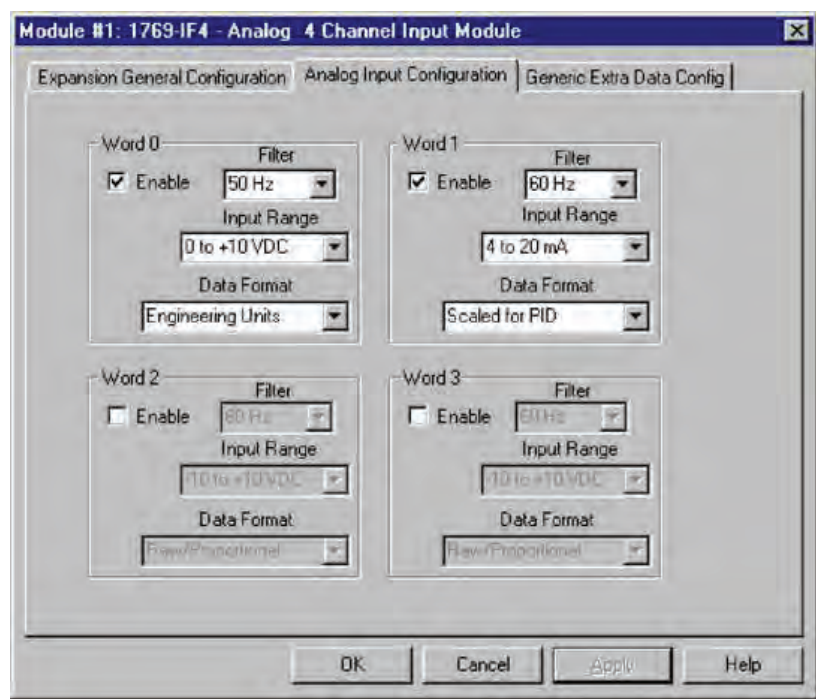

Fig.7.Analog channel configuration

In this paper, Allen-Bradley Micrologix 1200 series $\mathrm{C}$ PLC is used to perform required control actions. Here, PLC analog and digital modules both are required to implement this project and signal conditioning circuits are also needed to drive field devices. Six digital input terminals are required to interface master start and stop buttons of electrical machines [8]. Here two buttons are used for start and stop of rotary KILN, two switches for coal conveyor and two more switches for iron ore 
conveyor belt. Two analog input terminals are required to interface $\mathrm{K}$ type thermocouples and two analog outputs are required to drive conveyor DC motors. If indication is required then more digital terminals are required.

\section{Operational procedure}

This paper describes the control of DRI process with the aided of PLC. In this process the iron ore is converted into sponge iron at different temperature ratings with respect chemical reactions [8]. The quality of product depends on temperature ratings at the KILN junctions. So, temperature has to be controlled in order to get high quality of product. Whenever process is started then the temperature at the junctions will be measured by thermocouples and which are interfaced to PLC analog input modules, this value is calibrated into temperature degree Celsius and displayed on SCADA screen.

PLC PID controller sends control signals to coal conveyor and blower fan by considering junction temperature in order to maintain desired temperature at the KILN junctions. Here, we need to develop the program for this control operation using PLC PID functions by considering controller tuning methods. If the temperature exceeds the desired values then the PLC sends the control signals to blower fan and coal conveyor then the speed of conveyor belt on blower fan either decreased or increased until reached the desired value. The controlling operations can be taken by user from the SCADA system and we can also monitor the status of process on SCADA screen. The designed project is shown in the below fig. 8 .

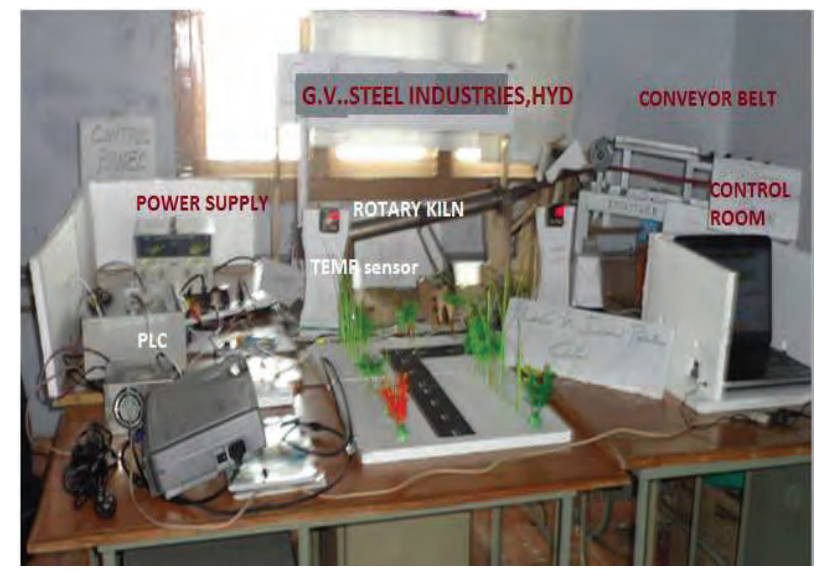

Fig.8.Designed project (Hardware equipment)

\section{E. Tuning of controller}

Tuning is the process of selecting the best parameters of controller. In this paper we are using Ziegler-Nichols PID tuning method. This is the methods for finding proper values of $\mathrm{Kp}$, Ti and $\mathrm{Td}$.

While tuning is going, mainly four parameters should be considered, these are settling time, off set, good stability and overshoot. Here $\mathrm{Kp}, \mathrm{Ki}$ and $\mathrm{Kd}$ values should be tuned until to get the good stability and response of the process is shown in Fig.9. If the damping ratio is very high then the response of the system is very slow, so which takes a lot of time to reach the set point. If the damping ratio is zero then it produces continuous oscillations then the system will go to instability conditions, which causes to damage of final control element [9]. If the damping ratio is less than 1 then it produces under damped response, which is very speed response but poor stability. If the damping ratio is 0.5 then it has acceptable stability and medium fastness.

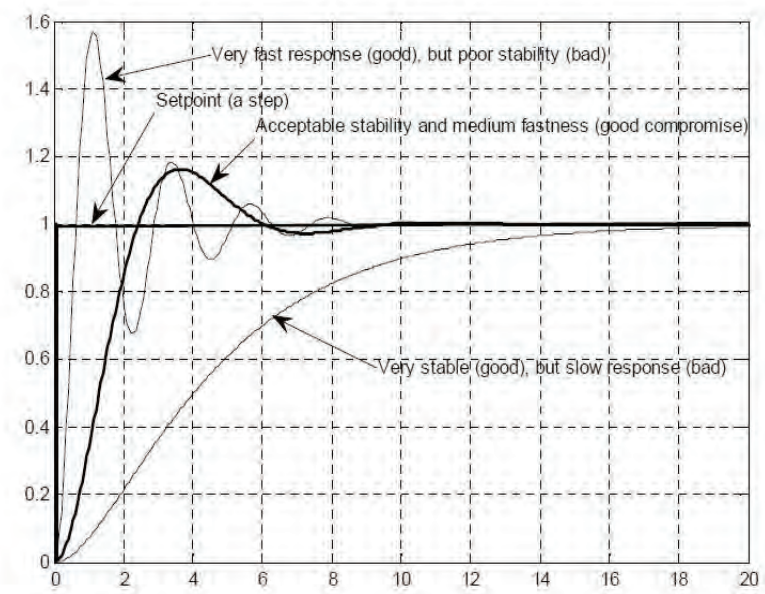

Fig 9: In controller tuning we want to achieve this compromise: Acceptable stability and medium fastness of response

\section{The Ziegler-Nichols’ Pid Tuning Procedure}

In this paper, DC motor to be controlled by the PLC-PID controller [9], so PID controller parameters have been tuned in order to get good response of the system. To find the best parameters of the controller The ZieglerNichols' closed loop method is based on experiments executed on an established control loop (a real system or a simulated system), shown in Fig. 10.

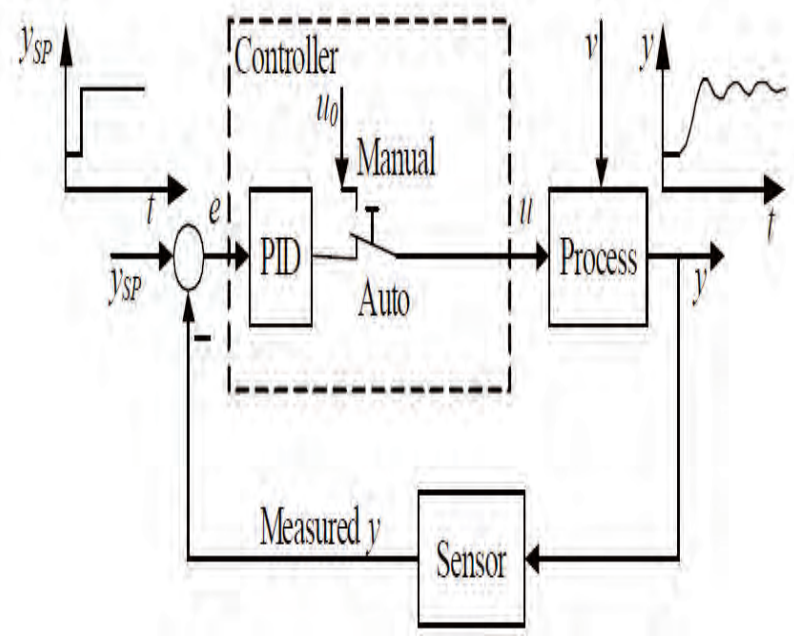

Figure.10: closed loop The Ziegler-Nichols' tuning method

The tuning procedure is as follows:

This method is primarily experimental \& uses real process data from the systems response. This method was developed by Ziegler \& Nichols, which is based on frequency response analysis. Unlike process reaction curve method which uses data from the Open loop response of a 
system, the Ziegler - Nichols Technique is a closed loop procedure.

In this method, first, bring the process to the desired operational level and then, use proportional control only with the feedback loop closed by vending the proportional gain until the system oscillates continuously \& let that proportional gained $\mathrm{Kc} \&$ period of Oscillations as $\mathrm{Pu}$. By using the following recommended settings for feedback. Controller given by Ziegler \& Nichols calculates the controller settings.

Table2: tuning parameters for P, PI, PID controllers

\begin{tabular}{|c|l|l|l|}
\hline Controller & \multicolumn{1}{|c|}{$\mathrm{K}_{\mathrm{c}}$} & \multicolumn{1}{|c|}{$\mathrm{T}_{\text {int }}$} & $\mathrm{T}_{\text {Der }}$ \\
\hline $\mathrm{P}$ & $\mathrm{K}_{\mathrm{c} / 2}$ & & \\
\hline $\mathrm{PI}$ & $\mathrm{K}_{\mathrm{c} /} / 2.2$ & $\mathrm{P}_{\mathrm{u}} / 1.2$ & \\
\hline $\mathrm{PID}$ & $\mathrm{K}_{\mathrm{c}} / 1.7$ & $\mathrm{P}_{\mathrm{u} /} 2$ & $\mathrm{P}_{\mathrm{u}} / 8$ \\
\hline
\end{tabular}

A. Input and output configuration with RS- Logix 500 After the completion of PLC programming, before going to dumping or downloading. We should read $\mathrm{I} / \mathrm{O}$ configuration of PLC. Once read I/O configuration then automatically detects PLC, which is interfaced to PC [RSLogic 500]. Which makes a path to dumping the programming to PLC. Here configuring is done automatically or manually. Once you open RS Logic 500 and then make double click on read I/O configuration then automatically shows different series of PLCs after that we need to select PLC series which has been interfaced to PC. After that write PLC programming into selected PLC and then go to RUN mod to monitor the status of I/O devices.

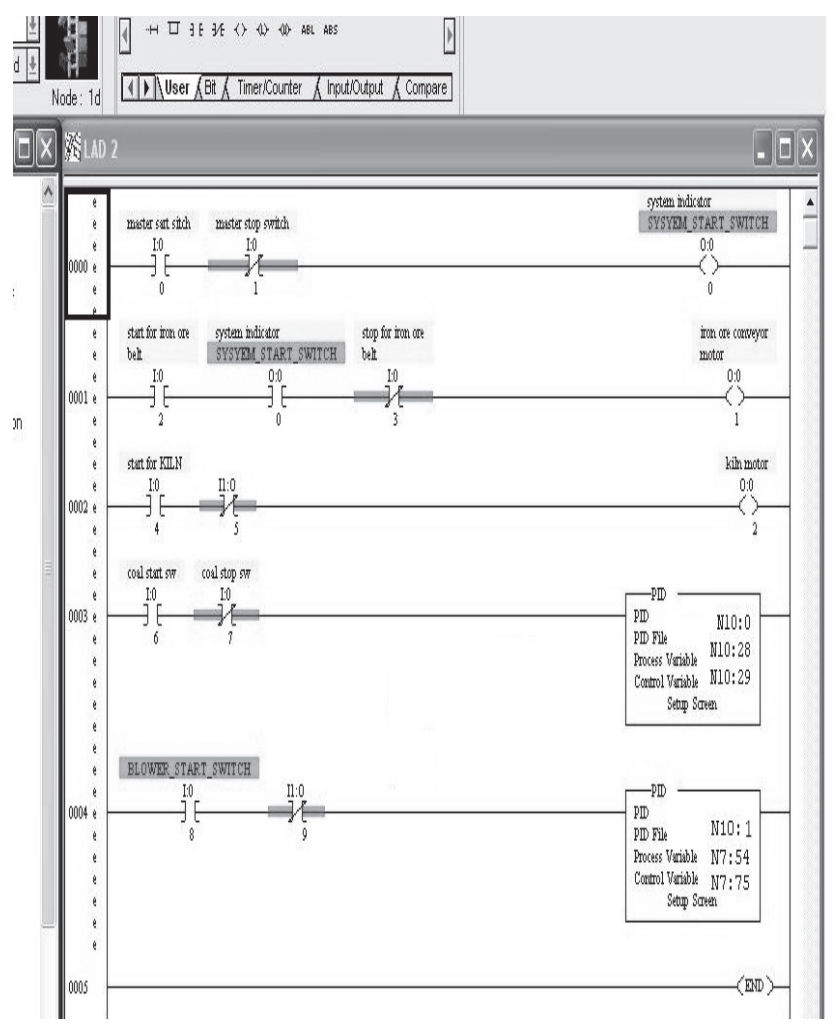

Fig.11.PLC program for the project
The following instructions are the desired PLC programming instructions to write the program [1].

\author{
1. Normally open \\ 2. Normally closed \\ 3. PLC PID function \\ 4. Arithmetic Functions \\ Addressing format \\ Input->I: 0/3, I: 0/2, I: 0/5, I: 1/0, I: 1/3 \\ Output->O: 0/0, O: 0/1, O: 1/0, O: 2/4
}

\section{SCADA AND RESUltS}

The status of field devices can be monitored and controlled by SCADA system [5]. In view of this Field devices and SCADA is interfaced through the PLC. RS View32 SCADA is used in this project.

Follow the steps below to link up PLC with RS VIEW32:

1. Go to Start and open RS VIEW SCADA software.

2. Click on File and create a new project by clicking on New. After creating a new project, the options available in the project manager

3. Go to system and double click on channel. In the Channel editor, select a channel and assign the appropriate network type to it as shown in figure.12. In the Primary Communication driver field [10], assign a driver to the channel. If you do not have drivers loaded, click None Loaded. For example, select DH-485as the network type, select the Primary Communication Driver as AB-DF1-1 and select the check box primary for active driver. 


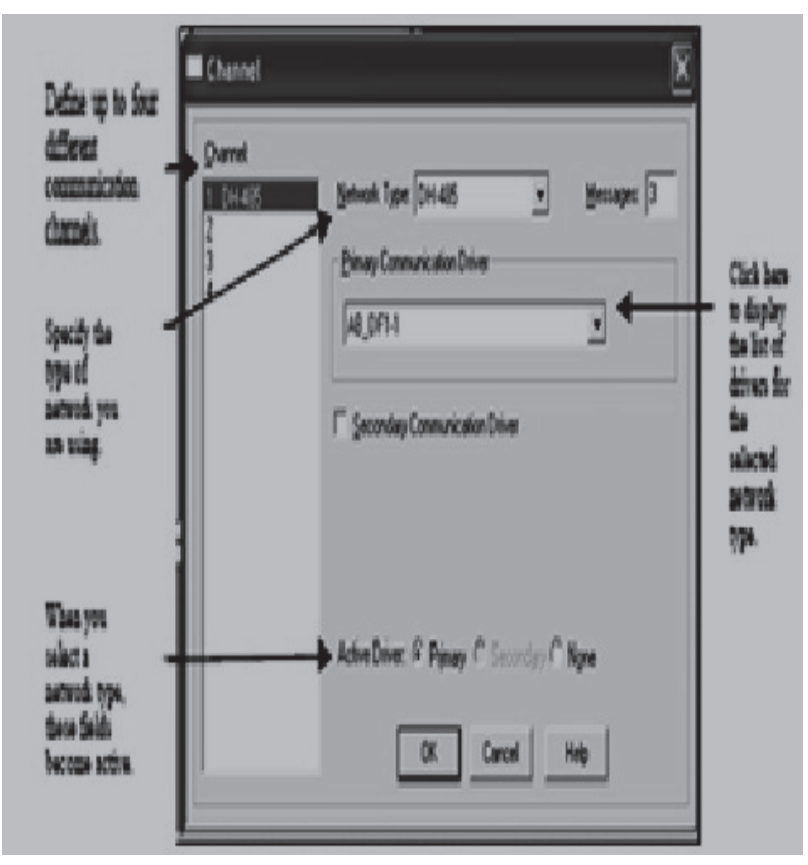

Figure 12: Configuring Channel for Direct Driver Communication

4. Create nodes according to the number of inputs and outputs required in the program. For each node assign the station by clicking the station browse option. The figure .12 shows the typical dialog box for creating nodes.

5. Accept after creating each node and click next so as to add each and every node.

6. Go to tag database after completing the nodes.

7. There are two ways by which tags can be created. The first way is in the Tag Database editor to Create tags [10]. For each tag, select Device as the data source and assign the nodes and scan classes that you have defined. The tag database dialog box is shown in figure 13 .

8. Now in tag database, add the name, type and security of tags one by one. Choose either device or memory depending on for what we are creating the tags.

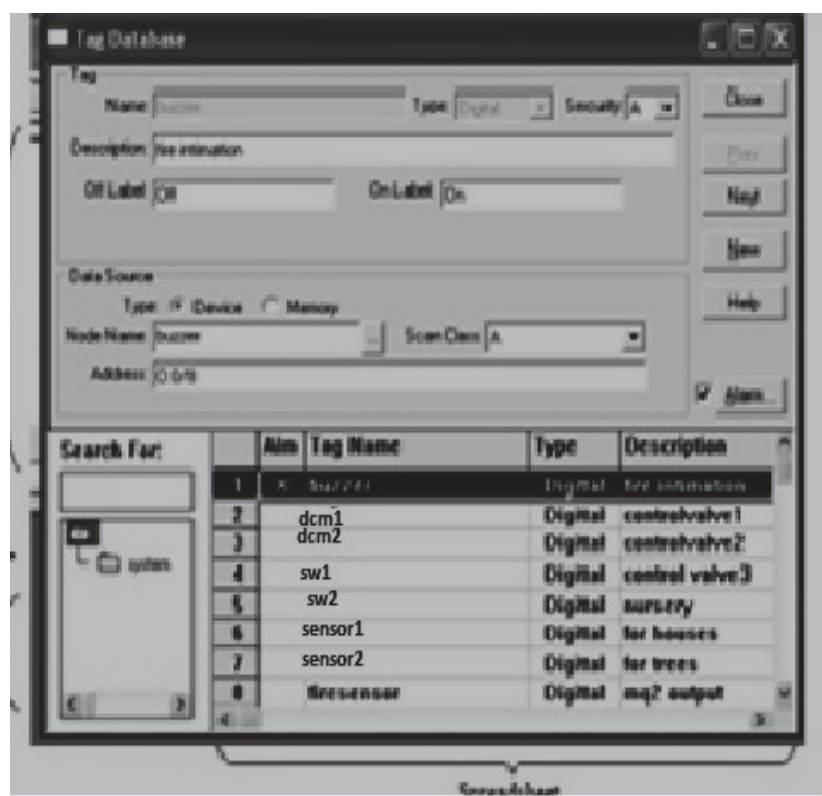

Figure 13: Creating tags using Tag Database

9. If device is chosen i.e., if we are taking the field devices and connecting to PLC then we need to choose device and give proper addressing format with its scan class and attach them to specific node.

10. After each tag, presses accept to save and then next to proceed to other tags.

11. Go to graphics and open display. Create the inputs and outputs in the white display with the help of library.

12. Right click on each element and select animation and supply visibility, color, rotation, fill etc. as required by the user. For each animation, tags have to be imported by browsing from the tags.

13. After the animations are done for each and every object according to the program, save the display and it can be retrieved from the library again.

14. After saving, run both the PLC ladder program [10] and test $u$ run for the SCADA screen created. We see that the current state of the inputs and output buttons or field devices is reflected on the screen at every instance.

SCADA results: Open the project file in the Rs View 32 software [5] then click on the then select the project file which is already designed. This page explains that it is a home screen for total project. This is shown in Fig.14. 


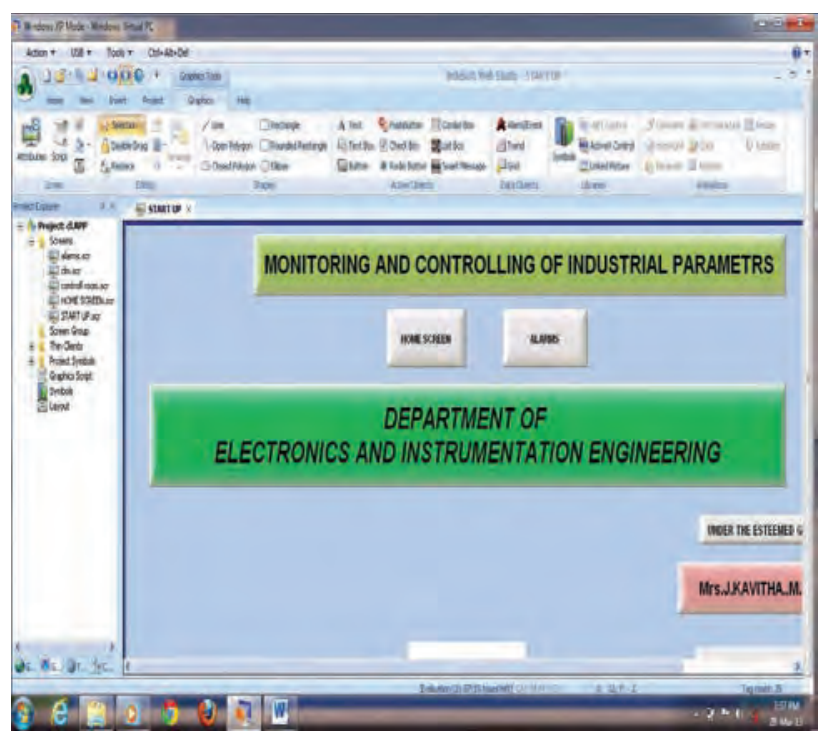

Fig .14.SCADA Home screen of the project.

Then click on the home page then a wizard is open as follows. It describes that this is the complete animated representation of total project is shown in fig. 15 . .Hence the total process can be monitored and controlled here.

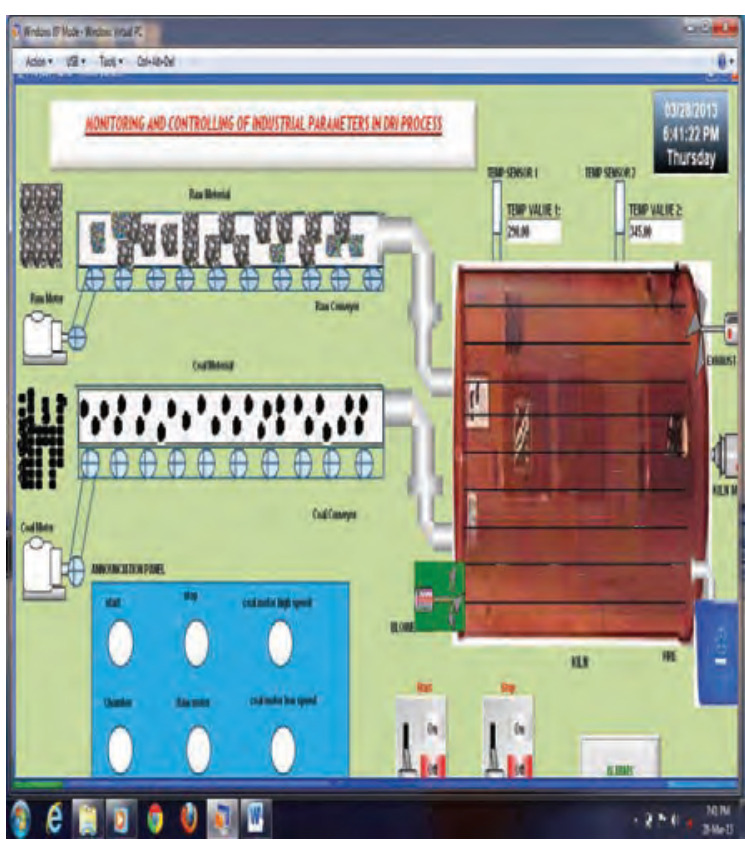

Fig.15.Total project representation through SCADA

\section{CONCLUSIONS}

The use of SCADA Based Automatic Direct Reduction of Iron (DRI) Process Using Allen-Bradley PLC is an advantage over the traditional control systems as the monitoring and controlling can be done without human intervention. Quality of production of iron can be increased by this automation process. This automatic process linearly changes the field parameters (like feed rate of raw material, inlet and outlet pressures) with respect to desired product quality.

\section{REFERENCES}

[1]. Nabil Daoud talka Quatar steel company limited,"Utilization of Sponge Iron in Electric Arc Furnace",These paper was originally presented at AISU's $2^{\text {nd }}$ electric furnace symposium in Damascus, Syria, Oct $18-20,1988$

[2]. Joanna Marie M. Baroro, Melchizedek I. Alipio, Michael Lawrence T. Huang, Teodoro M. Ricamara and Angelo A. Beltran Jr. "Automation of Packaging and Material Handling Using Programmable Logic Controller" International Journal of Scientific Engineering and Technology ,ISSN: 2277 - 1581, Volume No. 3, Issue No. 6, pp: 767 - 770,June 2014.

[3]. A Reis and John. W .Webb Ronald, Programmable Logic Controllers - Principles and Applications, Fourth edition, Prentice Hall Inc., New Jersey, 1998

[4]. K. Gowri Shankar " Control of Boiler Operation using PLC - SCADA, Proceedings of the International Multi Conference of Engineers and Computer Scientists, Vol-II ,IMECS 2008, Hong Kong, 19-21 March, 2008.

[5]. D.Roy Choudhury and Shail B Jain, "Linear Integrated Circuits", $2^{\text {nd }}$ Edition, New Age International (P) Limited, Publisher.pp:117-126.

[6]. Maha M. Lashin "Different Applications of Programmable Logic Controller (PLC)", International Journal of Computer Science, Engineering and Information Technology (IJCSEIT), Vol. 4,No. 1, February 2014.

[7]. Prof. Burali Y. N "PLC Based Industrial Crane Automation \& Monitoring" International Journal of Engineering and Science ISSN: 2278-4721, Vol. 1, Issue 3,PP 01-04, Sept 2012.

[8]. Dr. Md.Fakhruddin Ansari "Design and Implementation of SCADA Based Induction Motor Control". Journal of Engineering Research and Applications ISSN: 2248-9622, Vol. 4, Issue 3, pp.05-18, March 2014

[9]. Sk Hasan Hafizul Haque, H. M. Imran Hassan and S. M. Abul Hossain "Comparison of Control System Using PLC \& PID”, University of Bridgeport, USA. ASEE 2014 Zone I Conference, April 3-5, 2014.

[10]. Santosh B .Belekar,Abjitth A.desai, Megaraj H .Parit "PLC and SCADA based Distribution and Monitoring and Control" Multidisciplinary Journal of Research in Engineering and Technology Volume 1, Issue 1, pp. 105110, April 2014 